DE

M E D I C I N A

T R O P I C A L

$\mathrm{DE}$

SÃO PAULO

JOURNAL OF THE SÃO PAULO INSTITUTE OF TROPICAL MEDICINE

(1) Clinical Centre of Serbia, Clinic for Infectious and Tropic Diseases, Belgrade, Serbia

(2) University of Belgrade, School of Medicine, Belgrade, Serbia

(3) Clinical Centre of Serbia, Clinic for Pulmonology, Belgrade, Serbia

(4) University of Sassari, Department of Biomedical Sciences, Sassari, Italy

Correspondence to: Aleksandra Barac. Clinical Centre of Serbia, Clinic for Infectious and Tropic Diseases, Bulevar Oslobodjenja 16, 11000, Belgrade, Serbia.

E-mail: aleksandrabarac85@gmail.com

Received: 6 November 2016

Accepted: 9 March 2017

\section{Complications of chronic necrotizing pulmonary aspergillosis: review of published case reports}

\author{
Aleksandra Barac ${ }^{1}$, Tatjana Adzic Vukicevic ${ }^{2,3}$, Aleksandra Dudvarski Ilic ${ }^{2,3}$, \\ Salvatore Rubino ${ }^{4}$, Vladimir Zugic ${ }^{2,3}$, Goran Stevanovic ${ }^{1,2}$
}

\section{ABSTRACT}

Chronic necrotizing pulmonary aspergillosis (CNPA), a form of chronic pulmonary aspergillosis (CPA), affects immunocompetent or mildly immunocompromised persons with underlying pulmonary disease. These conditions are associated with high morbidity and mortality and often require long-term antifungal treatment. The long-term prognosis for patients with CNPA and the potential complications of CNPA have not been well documented. The aim of this study was to review published papers that report cases of CNPA complications and to highlight risk factors for development of CNPA. The complications in conjunction associated with CNPA are as follows: pseudomembranous necrotizing tracheobronchial aspergillosis, ankylosing spondylarthritis, pulmonary silicosis, acute respiratory distress syndrome, pulmonary Mycobacterium avium complex (MAC) disease, superinfection with Mycobacterium tuberculosis, and and pneumothorax. The diagnosis of CNPA is still a challenge. Culture and histologic examinations of bronchoscopically identified tracheobronchial mucus plugs and necrotic material should be performed in all immunocompromised individuals, even when the radiographic findings are unchanged. Early detection of intraluminal growth of Aspergillus and prompt antifungal therapy may facilitate the management of these patients and prevent development of complications.

KEYWORDS: Chronic necrotizing pulmonary aspergillosis. Complications. Aspergillus.

\section{INTRODUCTION}

Aspergillus infection of the lung manifests in a wide spectrum of conditions ranging from saprophytic colonization to hypersensitivity reactions to necrotizing pneumonia with angioinvasion ${ }^{1}$. In the saprophytic form of the disease, aspergillomas establish themselves in pre-existing cavities. The chronic cavitary form of aspergillosis, which produces a slowly progressive infiltrate that may include mycetoma, was proposed in 1981 as a new category of semi-invasive pulmonary ${ }^{2}$ or chronic necrotizing pulmonary aspergillosis $(\mathrm{CNPA})^{3}$. CNPA is a form of chronic pulmonary aspergillosis (CPA), often presenting with rapid progressive infection ( $<3$ months) in moderately immunocompromised patients, which should be managed as invasive aspergillosis ${ }^{1}$. Aspergillus has a propensity to invade pulmonary blood vessels, resulting in pulmonary infarction and subsequent development of necrotic cavities and bronchus dilatation ${ }^{4}$. Lung conditions affecting the pulmonary defense system, such as previous surgical resection, ionizing radiation therapy, or chronic obstructive pulmonary disease (COPD), allow Aspergillus to penetrate the lung through enzyme secretion despite the absence of cavities ${ }^{5}$. In addition to COPD, other conditions commonly associated with CNPA include previous pulmonary 
tuberculosis (TB), atypical Mycobacterium tuberculosis infection, cystic fibrosis, pulmonary infarction, pulmonary fibrosis, previous surgery, and pneumoconiosis ${ }^{6-8}$. Other conditions that may be associated with the development of CNPA include lung cancer, stage III or IV fibrocystic pulmonary sarcoidosis, and silicosis ${ }^{6-8}$. Other immunosuppression conditions such as diabetes mellitus, malnutrition, alcoholism, connective tissue diseases, and prolonged corticosteroid therapy also increase the risk for development of $\mathrm{CNPA}^{1,2}$. Complications of CNPA usually occur because of leakage of air from an air-filled lung cavity into the pleural space. These conditions are associated with high morbidity and mortality and often require long-term antifungal treatment ${ }^{9}$. The long-term prognosis for patients with CNPA and potential complications of CNPA have not been well documented. The aim of this study was to review published papers reporting cases of CNPA complications and to highlight risk factors for development of CNPA.

\section{Pseudomembranous necrotizing tracheobronchial aspergillosis and CNPA}

The Oh et al. ${ }^{10}$ reported a case of pseudomembranous necrotizing tracheobronchial aspergillosis in an immunocompetent host. They reported a rare case of Aspergillus pseudomembranous tracheobronchitis without an invasion of the pulmonary parenchyma that developed in an immunocompetent patient ${ }^{10}$. Aspergillus tracheobronchitis was confined, at least initially, to the airways with only a superficial mucosal invasion, and the diagnosis was delayed because of non-specific signs, symptoms, and lack of radiographic abnormalities ${ }^{11}$. Pseudomembranous necrotizing tracheobronchial aspergillosis has been reported recently as an uncommon form of airway invasive aspergillosis in immunocompromised patients ${ }^{10,12-15}$. In a review of the literature on Aspergillus tracheobronchitis, including all forms of tracheobronchial involvement, reports indicated that the disease was progressive and fatal in about $40 \%$ of cases ${ }^{10,13-15}$. This was apparently a lethal variant of tracheobronchial aspergillosis with a markedly higher mortality rate and more parenchymal lung involvement (65\%) compared with all cases of Aspergillus tracheobronchitis $(45 \%)^{10,15}$. It presents an extensive transmural necrotizing bronchitis with pseudomembrane formation that develops in immunocompromised patients, and it is usually characterized by a limited extension of inflammation and hyphae into the peribronchial tissue, without an invasion of the pulmonary parenchyma ${ }^{10,12}$. Involvement of the bronchial tree can be invasive, leading to a pseudomembranous tracheobronchitis with extensive bronchial hemorrhage and invasion of the bronchial walls and vessels. In the literature review, we found reports of 22 cases with this variant of tracheobronchial involvement by Aspergillus ${ }^{12-15}$. There are published only five reports of this disease developing in an immunocompetent or mildly immunocompromised patient. The first case revealed cutaneous anergy and lymphopenia involving $\mathrm{T}$ cells and NK cells, related to a recent influenza A infection ${ }^{16}$. In the second case, there were "grayish" mucus plugs in several segmental bronchi on autopsy and focally limited extension into the adjacent lung parenchyma ${ }^{17}$. The third case revealed the presence of fungal invasion through the bronchial wall into the adjacent lung parenchyma and pulmonary arteries ${ }^{17}$. The fourth case was a 70-year-old man with many risk factors, including corticosteroid use. The fifth case was a 66-year-old with underlying COPD ${ }^{17}$. This patient had no risk factors associated with an immunocompromised state, and had no invasion of the parenchyma, or atelectasis, but his condition rapidly progressed to severe $\mathrm{CO} 2$ retention and respiratory failure. Pseudomembranous necrotizing tracheobronchial aspergillosis should be considered as potential complication of CNPA even in an immunocompetent host $\mathrm{t}^{10,15,16}$.

\section{Ankylosing spondylarthritis and CNPA}

Fibro-bullous disease is a late complication of ankylosing spondylarthritis ${ }^{17,18}$. Its radiologic features frequently mimics tuberculosis or excavated neoplasm. About 50 to $65 \%$ of patients with ankylosing spondylarthritis have chronic Aspergillus colonization of their airways ${ }^{17}$. Pulmonary aspergillosis infection occurs in 10 to $30 \%$ during evolution. Pontier et al. reported two cases of patients with spondylarthritis who developed chronic necrotizing pulmonary aspergillosis ${ }^{17}$. Diagnosis was based on serology, mycologic examination of expectoration and in one case, on histology obtain by transbronchial biopsies. Pulmonary complications due to Aspergillus species frequently reveal associated fibro-bullous disease in patients with ankylosing spondylarthritis. The fibroblastic disease is often asymptomatic and, as in the reported cases, its clinical revelation is often linked to a complication (bacterial or fungal infections, tuberculosis, pneumothorax, etc.). In one case, it was associated with unilateral, pseudo-tuberculosis, with a preferential apical location and pleural involvement. In cases with ankylosing spondylarthritis and CNPA, there is usually bilateral and asymmetric pachypleuritis ${ }^{17-19}$. This is a rare complication of spondylitis in about $1.3 \%$ of patients ${ }^{18}$, with male predominance and it is estimated that it is revealed on average 17 years after the spinal signs. There 
is no specific predictive factor (HLA B27 antigen positive in $50 \%$ of cases $)^{18}$. The two patients reported by Pontier et al. ${ }^{17}$, had no previously known pulmonary pathology ${ }^{17}$. At the anatomo-pathologic level, lesions of inter-alveolar fibrosis with hyaline fibrosis and non-specific lymphocytic and macrophage infiltrates were found ${ }^{17}$. The origin of this fibrosis is currently unknown but several hypotheses have been suggested: an inflammatory fibrosis aggravated or caused by repeated infectious episodes, a hypoventilation of the apexes favored by the restrictive syndrome and systemic inflammatory attack comparable with the involvement of other organs ${ }^{17-19}$. The evolution can be towards chronic respiratory insufficiency.

Semi-invasive pulmonary aspergillosis or CNPA occurs in $10-30 \%$ of cases of fibroblastic disease. It is a frequent mode of revelation ${ }^{17-19}$. Unlike aspergilloma, there is no preexisting cavity, and no walls are built. It is probably favored by hypoventilation of the vertices during spondylitis. It may develop into fibroblastic sequelae. The question arises as to whether fibroblastic disease is a sequela of semi-invasive pulmonary aspergillosis or pre-existing in asymptomatic form. In both cases, semi-invasive aspergillosis is manifested as severe broncho-pneumonia, resistant to antibiotics, and potentially fatal ${ }^{17}$. The general clinical sign predominates and distinguishes it from aspergilloma. Pontier et al. ${ }^{17}$ describe the acute form, evolving in a few weeks towards pneumonic block and signs of suppuration, and sub-acute form, with a pseudo-tuberculous picture evolving towards necrosis. In the cases presented by Pontier et al. ${ }^{17}$, a radio-clinical picture of intra-cavitary aspergillosis was demonstrated after favorable response to antifungal treatment. Although cases of isolated aspergilloma have been described in patients with ankylosing spondylitis with fibroblastic lesions ${ }^{17-19}$, in the two cases published by Pontier et $a l .{ }^{17}$, were probably a result of low-lying progression of semi-invasive aspergillosis previously incompletely controlled by medical treatment.

\section{Pulmonary silicosis and CNPA}

Blanco et al..$^{20}$ reported three cases with a long history of exposure to silica dust with pulmonary aspergillosis complicated bu progressive massive fibrosis (PMF) ${ }^{20}$. Pneumoconiosis, which causes deterioration of local immunity, is less frequent, limited to $2 \%$ and $3 \%$ of $\operatorname{cases}^{21,22}$. The three cases presented by Blanco et al. ${ }^{20}$ presented had a history of tuberculosis, which was variably described in the literature (between $11 \%$ and $93 \%)^{21,22}$. Frequent treatment with steroids in the first patient and a history of alcoholism in the second patient probably contributed to the predisposition for $\mathrm{CNPA}^{20}$. On the other hand, all three had been active smokers ${ }^{20}$, a history referred to in $53 \%$ of the patients reported by Nam et al..$^{23}$ but not mentioned in the other series. Compared with Nam et al. ${ }^{23}$ reports, in which the youngest patient was 48 years old and only $20 \%$ had large opacities, patients reported by Blanco et al. ${ }^{20}$ were younger and PMF was observed in all three cases. These patients usually present with fever, cough and weight loss, and less frequently dyspnea or chest pain. The three patients reported by Blanco et al..$^{20}$ had hemoptysis and mycetoma ${ }^{20}$, which is reported in $49 \%$ and $90 \%$ of cases in the literature ${ }^{21-23}$. Radiologically, the presence of infiltrates in the upper lobes, cavitation, nodular opacities, and pleural thickening are the most common findings that coincide with silicosis in patients with $\mathrm{CNPA}^{20-23}$.

CNPA can be serious, fatal if untreated, and may be accompanied by acute respiratory distress syndrome ${ }^{23-24}$. CNPA can also play a relevant role in the mortality of patients with silicosis. The study by Iossifova et al. ${ }^{25}$ showed that people with CNPA and silicosis are almost 10 times more likely to die than people without silicosis. In addition, in young patients, lung transplantation could be contraindicated, as shown in the first case reported by Blanco et al. ${ }^{20}$.

Given the high mortality, the diagnosis of CNPA in patients with silicosis should be considered as soon as possible, especially if there are any signs of new infiltrates, mycetomas, and anamnestic data of active smoking or a history of $\mathrm{TB}^{20-24}$.

\section{Acute respiratory distress syndrome and CNPA}

Yano $^{24}$ published a report of a patient with acute respiratory distress syndrome (ARDS) who died because of CNPA. This patient had pulmonary fibrosis of unknown cause with a right upper bulla. Mycetoma in the bulla, invaded the tissue adjacent to the bulla, as the wall of the right upper bulla became thicker with surrounding lung infiltration ${ }^{24}$. This patient developed ARDS because of CNPA after Aspergillus invaded these tissues leading to fatal respiratory failure. The patient had a cerebral accident 6 days after respiratory failure, probably as a result of septic emboli accompanied with CNPA. Depending on the size of the emboli, nuclear magnet resonance (NMR) findings may vary from major arterial branch infarction to multiple small abscesses located at the gray-white matter junction, secondary to occlusion of small arteries and arterioles ${ }^{25,26}$. Small abscesses are accompanied by surrounding oedema and mass effect, which is well demonstrated on NMR ${ }^{1}$. One day before the first cerebral accident in case reported by Yano $^{24}$, the percentage of serum eosinophils increased to $13 \%$; after the first cerebral attack, the increased percentage 
of eosinophils persisted at around $13-22 \%$. This peripheral eosinophilia may reflect activation of the lung aspergillus lesion. It is important to consider that ARDS may occur in cases of CNPA and that peripheral eosinophilia might forebode worsening of $\mathrm{CNPA}^{24-28}$.

\section{Pulmonary Mycobacterium avium complex disease and CNPA}

Pulmonary aspergilloma is an important complication occurring in cavities remaining after pulmonary $\mathrm{TB}$, and it has been reported as a complication in $15 \%$ of patients with sequelae of pulmonary $\mathrm{TB}^{28-30}$. Although the most frequent underlying disease with pulmonary aspergillosis was pulmonary $\mathrm{TB}^{29}$, there are no detailed reports regarding the formation of pulmonary aspergilloma including CNPA with a fungus ball in a cavity formed by pulmonary Mycobacterium avium intracellular complex (MAC). Kobashi et al..$^{30}$ reported a case of CNPA in which a fungus ball appeared in a growing cavity transitionally formed by pulmonary MAC, during a follow-up period after treatment ${ }^{30}$. The most frequently reported underlying disease in cases of pulmonary aspergilloma including CNPA is sequela of pulmonary TB (25-72\%), secondary to a pre-existing intrapulmonary cavity such as a pulmonary cyst $^{29,31,32}$. There have been a few reports of superinfection by Aspergillus spp. of pulmonary lesions caused by Mycobacterium xenopi ${ }^{33,34}$. However, the frequency of nontuberculous mycobacteria (MAC, etc.) has recently increased among patients with pulmonary mycobacterial diseases, especially in industrialized nations ${ }^{29}$. In addition, there are many cases in previous reports in which the underlying disease of pulmonary aspergilloma including CNPA was a cavity formed by a pulmonary nontuberculous mycobacterial, rather than a cavity formed by pulmonary $\mathrm{TB}^{29-34}$. The clinical symptoms caused by pulmonary aspergilloma vary in accordance with the severity of the underlying pre-existing pulmonary disease, but a common characteristic has been hemosputum or haemoptysis ${ }^{29-34}$ The chief complaint in the case presented by Kobashi et $a l .^{30}$ was continuous fever, which suggests that the patient probably developed the condition, which suddenly destroyed the lung, as semi-invasive pulmonary aspergillosis or CNPA, rather than pulmonary aspergilloma. Denning ${ }^{31}$ also reported that patients with pulmonary aspergilloma with clinical symptoms such as fever and cachexia usually have undiscovered CNPA, rather than pulmonary aspergilloma. The patient presented by Denning ${ }^{31}$ had a mixed infection caused by A. niger and $M$. avium, although it has been commonly mentioned that $A$. fumigatus is the most frequent isolate from patients with CNPA. Kawamura et al..$^{30}$ and
Perfect et al. ${ }^{32}$ reported isolation rates of A. fumigatus of $75 \%$ and $80 \%$, respectively; the rates for A. niger were $9 \%$ and $10 \%$, respectively.

With regard to the laboratory findings, it has been reported $^{32-35}$ that tumor markers (carcinoembryonic antigen, squamous cell carcinoma antigen, carbohydrate antigens sialyl Lewis, and sialyl SSEA-1) are often increased in several benign diseases such as pulmonary mycobacterial diseases, and the percentage increase of these tumor marker is $15-62 \%$. Histologically, these tumor markers were expressed in mucous cells of the bronchial gland and the surface of bronchiolar surface epithelium cells ${ }^{29,31-33}$. Consequently, it is suspected that serum tumor markers are increased in cases of hyperplasia of bronchiolar epithelium cells or mucous cells of bronchial gland, because of the existing chronic respiratory infection with bronchiectasis caused by pulmonary MAC ${ }^{29,31-33}$. The case presented by Kobashi et al. ${ }^{30}$ serves as a reminder that pulmonary nontuberculous mycobacterial disease has become one of the underlying pulmonary diseases complicated by CNPA. In order to detect fast CNPA with a fungus ball as a complication, it is important to perform periodic radiological examinations (chest computed tomography $[\mathrm{CT}]$ ), serological examinations (anti-Aspergillus antibody) and sputum culture examinations during the follow-up period of an underlying respiratory disease $\mathrm{e}^{29-34}$.

\section{Superinfection by Mycobacterium tuberculosis and CNPA}

Although aspergillosis is commonly found in old tuberculous cavities, pulmonary aspergillosis is rarely complicated by active pulmonary $\mathrm{TB}^{34,35}$. Kohno et al. ${ }^{36}$ presented a rare case of CNPA complicated by active pulmonary TB. The case is unusual because the necrotic granuloma of aspergillosis contained a few acid-fast bacilli in the necrotic foci. Colonization of pre-existing TB pulmonary lesions by Aspergillus species is well known; however, superinfection by Aspergillus of a cavity already infected by Mycobacterium is rare ${ }^{34}$. Binder et $a l .^{3}$ reported a case of CNPA with a moderate number of acid-fast bacilli, but commented that this might have been colonization by non-tuberculosis mycobacteria. Because the necrotic granuloma consisted mainly of Aspergillus hyphae, it is more likely that aspergillosis was the main lesion with M. tuberculosis superinfection. It is impossible to completely rule out the possibility that this case was an instance of aspergillosis invading a healed tuberculous lesion, but the pathologic features of CNPA are unique and characteristic $^{34}$. Superinfection of CNPA with Xanthomonas maltophilia has also been reported, but it is rare ${ }^{3}$. 


\section{Pneumothorax and CNPA}

Adzic-Vukovic et al. reported a rare and unusual case of pneumothorax in an immunocompetent patient with $\mathrm{CNPA}^{35}$. To our knowledge, this is the first reported case in Europe. A 40-year old woman, with body mass index less than $15 \mathrm{~kg} / \mathrm{m}^{2}$ and high-grade fever developed symptoms of acute onset of breathlessness, dry cough, and chest pain on the left side. The left side of the chest was hyper-resonant and breath sounds were weak. Laboratory results excluded common variable immune deficiencies, but on the other hand, the patient could not be considered as clinically immunocompetent because she had end-stage COPD and cachexia. Radiography showed a left-sided pneumothorax with contralateral cavitation and an intercostal drainage underwater seal was applied (Figure 1). Four days later, CT scan of the thorax indicated the presence of possible necrotizing lesions bilaterally (Figure 1). Fiber-optic bronchoscopy was done and a tissue sample was taken. Serological analyses revealed a positive galactomannan index (2.19) in bronchoalveolar lavage (BAL), as well as a positive value for anti-Aspergillus $\mathrm{IgG} \mathrm{Ab}$ in serum $(280 \mathrm{UI} / \mathrm{mL})$. Patho-histologic analyses of the tissue sample confirmed the presence of fungal hyphae, and culture was positive for A. fumigatus (Figure 1). The patient responded positively to antifungal therapy; her general condition improved with rapid radiographic improvement in 2 weeks. During a follow-up period of 1 year, there was no disease regression ${ }^{35}$.

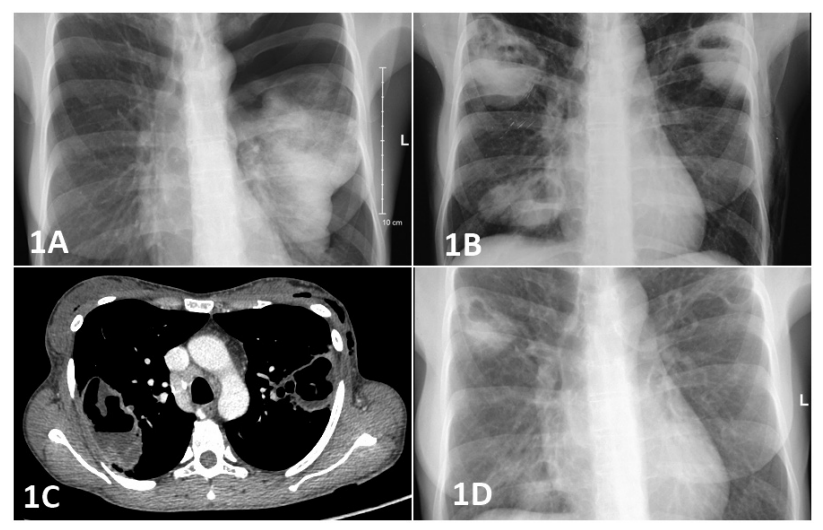

Figure 1 - 1A) Left sided pneumothorax with a contralateral cavitation; 1B) Bilateral cavity lesions; 1C) Computed tomography scan showed bilateral necrotising pulmonary lesions; 1D) Radiographic regression after two weeks of voriconazole treatment.

Pneumothorax as a result of rupture of aspergilloma into the pleural space in non-immunocompromised patients is extremely rare $^{8}$. Pneumothorax has been reported in granulocytopenic patients undergoing intensive cytotoxic therapy for hematologic malignancies ${ }^{8}$. In this case, the patient was immunocompetent according to the laboratory parameters, but clinically she was immunocompromised with end-stage COPD and cachexia ${ }^{35}$. Surprisingly, the patient developed pneumothorax as a complication of the rupture of aspergilloma into the pleural space. Long-term weight loss, low body mass index and untreatable end-stage COPD should be considered as possible cause of spontaneous pneumothorax in this patient. Pneumothorax increases both short-and long-term morbidity and mortality in patients with CNPA and causes significant deterioration of their quality of life. This case highlights that a diagnosis of CNPA should be considered in patients with end-stage COPD, a low body mass index, or patients who develop pneumothorax.

\section{CONCLUSION}

Diagnosis of CNPA is still a challenge. A chest radiograph is not helpful to the diagnosis for several reasons: (1) usually because of normal radiologic findings, intraluminal mycetomas may not be readily detectable, (2) Aspergillus may enlarge so rapidly that recent chest radiographs may not reveal atelectasis, and (3) the lung parenchyma is usually unaffected ${ }^{37}$. A chest CT scan is much more helpful in the early diagnosis and aids further diagnostic studies, such as bronchoscopies and open-lung biopsies. Although a CT scan is helpful in the diagnosis of airway aspergillosis, diagnosis of this specific disease cannot be made based on imaging features alone. Bronchoscopy, apart from providing specimens for microbiological examination, is a useful method for detecting infections with endobronchial manifestations ${ }^{37}$. It should be recommended as early as possible for diagnosis and to maintain airway patency, at a time when it is most feasible ${ }^{37-39}$. A presumptive diagnosis is made when the fungus is isolated from bronchial aspirates or BAL cultures, and a definitive diagnosis is made when there is histologic demonstration of invasion of the mucosa by hyphae ${ }^{37-39}$. Therefore, culture and histologic examinations of bronchoscopically identified tracheobronchial mucus plugs and necrotic material should be performed in all immunocompromised individuals, even when the radiographic finding are unchanged. Early detection of intraluminal growth of Aspergillus and prompt antifungal therapy may facilitate the management of these patients and prevent development of complications.

\section{ACKNOWLEDGEMENT}

The paper has been published with the support of the Ministry of Education, Science and Technology of the Republic of Serbia (Project No. III45005). 


\section{CONFLICT OF INTERESTS}

Authors declare there is no conflict of interests.

\section{REFERENCES}

1. Denning DW, Cadranel J, Beigelman-Aubry C, Ader F, Chakrabarti A, Blot S, et al. Chronic pulmonary aspergillosis: rationale and clinical guidelines for diagnosis and management. Eur Respir J. 2016;47:45-68.

2. Gefter WB, Weingrad TR, Epstein DM, Ochs RH, Miller WT. "Semi-invasive" pulmonary aspergillosis: a new look at the spectrum of aspergillus infections of the lung. Radiology. 1981;140:313-21.

3. Binder RE, Faling LJ, Pugatch RD, Mahasaen C, Snider GL. Chronic necrotizing pulmonary aspergillosis: a discrete clinical entity. Medicine (Baltimore). 1982;61:109-24.

4. Pittet D, Huguenin T, Dharan S, Sztajzel-Boissard J, Ducel G, Thorens JB, et al. Unusual cause of lethal pulmonary aspergillosis in patients with chronic obstructive pulmonary disease. Am J Respir Crit Care Med. 1996;154:541-4.

5. Kibbler CC, Milkins SR, Bhamra A, Spiteri MA, Noone P, Prentice HG. Apparent pulmonary mycetoma following invasive aspergillosis in neutropenic patients. Thorax 1988;43:108-12.

6. Attaway AH, Jacono F, Gilkeson R, Faress JA. Subacute invasive pulmonary aspergillosis associated with ankylosing spondylitis. Am J Respir Crit Care Med. 2016;193:572-3.

7. Peghin M, Ruiz-Camps I, Garcia-Vidal C, Cervera C, Andreu $\mathrm{J}$, Martin M, et al. Unusual forms of subacute invasive pulmonary aspergillosis in patients with solid tumors. J Infect. 2014;69:387-95.

8. Gupta PP, Fotedar S, Agarwal D, Magu S, Saini K. Pneumothorax: a rare presentation of pulmonary mycetoma. Ann Thorac Med. 2007;2:171-2.

9. Walsh TJ, Anaissie EJ, Denning DW, Herbrecht R, Kontoyiannis DP, Marr KA, et al. Treatment of aspergillosis: clinical practice guidelines of the Infectious Disease Society of America. Clin Infect Dis. 2008;46:327-60.

10. Oh HJ, Kim HR, Hwang KE, Kim SY, Ahn SH, Yang SH, et al. Case of pseudomembranous necrotizing tracheobronchial aspergillosis in an immunocompetent host. Korean J Intern Med. 2006;21:279-82.

11. Sayiner A, Kursat S, Toz H, Duman S, Onal B, Tumbay E. Pseudomembranous necrotizing bronchial aspergillosis in a renal transplant recipient. Nephrol Dial Transplant. 1999;14:1784-5.

12. Ahn MI, Park SH, Kim JA, Kwon MS, ParkYH. Pseudomembranous necrotizing bronchial aspergillosis. Br J Radiol. 2000;73:73-5.

13. Silveira F, Paterson DL. Pulmonary fungal infections. Curr Opin Pulm Med. 2005;11:242-6.
14. Kim JS, Rhee Y, Kang SM, Ko WK, Kim YS, Lee JG, et al. A case of endobronchial aspergilloma. Yonsei Med J. 2000;41: 422-5.

15. Clarke A, Skeleton J, Fraser RS. Fungal tracheobronchitis: report of 9 cases and review of the literature. Medicine (Baltimore). 1991;70:1-14.

16. Boots RJ, Paterson DL, Allworth AM, Faoagali JL. Successful treatment of post-influenza pseudomembranous necrotizing bronchial aspergillosis with liposomal amphotericin, inhaled amphotericin B, gamma interferon and GM-CSF. Thorax. 1999;54:1047-9.

17. Pontier S, Bigay L, Doussau S, Recco P, Lacassagne L, Didier A. Aspergillose pulmonaire nécrosante chronique et spondylarthrite ankylosante. Rev Mal Respir. 2000;17:683-6.

18. Peltier P, Germaud P, Martin M, Barrier J, Cottin S, Grolleau JY. Fibrose pulmonaire et pelvi-spondylite rhumatismale: à propos de 5 cas. Sem Hop. 1982;58:596-600.

19. Bourgeois P. Manifestations systémiques de la spondylarthrite ankylosante. In: Kahn MF, Peltier AP, Meyer O, Piette JC, éditeurs. Les maladies systémiques. $3^{\text {eme }}$ ed. Paris: MédecineSciences Flammarion; 1991. p.839-55.

20. Blanco JJ, Barcala FJ, Moure MA, Mao MC. Aspergilosis pulmonar necrotizante crónica como complicación de silicosis. An Sist Sanit Navar. 2011;34:109-14.

21. Hohl TM, Feldmesser M. Aspergillus fumigatus: principles of pathogenesis and host defense. Eukaryot Cell. 2007;6: 1953-63.

22. Denning DW, Riniotis K, Dobrashian R, Sambatakou H. Chronic cavitary and fibrosing pulmonary and pleural aspergillosis: case series, proposed nomenclature change, and review. Clin Infect Dis. 2003;37 Suppl 3:S265-80.

23. Nam HS, Jeon K, Um SW, Suh GY, Chung NP, Kim H, et al. Clinical characteristics and treatment outcomes of chronic necrotizing pulmonary aspergillosis: a review of 43 cases. Int J Infect Dis. 2010;14:e479-82.

24. YanoS.Acuterespiratory distress syndrome due to chronic necrotizing pulmonary aspergillosis. Intern Med. 2007;46:889-91.

25. Iossifova Y, Bailey R, Wood J, Kreiss K. Concurrent silicosis and pulmonary mycosis at death. Emerg Infect Dis. 2010;16:31820.

26. Parker JC Jr, Dyer MC. Neurologic infections due to bacteria, fungi, and parasites. In: Davis RL, Robertson DM, editors. Textbook of neuropathology. Baltimore: Williams \& Wilkins; 1985. p. 632-703.

27. Whiteman ML, Boman BC, Post MJ, Bell MD. Intracranial infection. In: Atlas SW, editor. Magnetic resonance imaging of the brain and spine. $2^{\text {nd }}$ ed. New York: Raven; 1996. p.729-32.

28. Alan MS, Elizabeth AO. Aspergillus syndrome, mucormycosis and pulmonary candidiasis. In: Fishman AP, editor. Fishman's pulmonary diseases and disorders. $3^{\text {rd }}$ ed. New York: McGrawHill; 1998. p.2265-8. 
29. Kawamura S, Maesaki S, Tomono K, Tashiro T, Kohno S. Clinical evaluation of 61 patients with pulmonary aspergilloma. Intern Med. 2000;39:209-12.

30. Kobashi Y, Yoshida K, Miyashita N, Niki Y, Matsushima T. Chronic necrotizing pulmonary aspergillosis complicated by a cavitary lesion caused by Pulmonary Mycobacterium-avium complex disease. Intern Med 2005; 44(3):246-50.

31. Denning DW, Pleuvry A, Cole DC. Global burden of chronic pulmonary aspergillosis complicating sarcoidosis. Eur Respir J. 2013;41:621-6.

32. Perfect JR, Cox GM, Lee JY, Kauffman CA, de Repentigny L, Chapman SW, et al. The impact of culture isolation of Aspergillus species: a hospital-based survey of aspergillosis. Clin Infec Dis. 2001;33:1824-33.

33. Johnston ID. Mycobacterium xenopi infection and aspergilloma. Tubercle. 1988;69:139-143.

34. Choudat D, Horreard P, Pretet S, Grosset J, Toty L. Superinfection by Aspergillus fumigatus of a pulmonary lesion caused by Mycobacterium xenopi. Tubercle. 1986;67:71-4.

35. Vukicevic TA, Dudvarski-Ilic A, Zugic V, Stevanovic G, Rubino
S, Barac A. Subacute invasive pulmonary aspergillosis as a rare cause of pneumothorax in immunocompetent patient: brief report. Infection. In Press 2017.

36. Kohno S, Hatano T, Yamada H, Maesaki S, Yasuoka A, Kaku $\mathrm{M}$, et al. Superinfection of chronic necrotizing pulmonary aspergillosis by Mycobacterium tuberculosis. Intern Med. 1992;31:540-3.

37. Patterson TF, Thompson GR 3rd, Denning DW, Fishman JA, Hadley S, Herbrecht R, et al. Practice guidelines for the diagnosis and management of aspergillosis: 2016 update by the Infectious Diseases Society of America. Clin Infect Dis. 2016;63:e1-e60.

38. Cadranel J, Philippe B, Hennequin C, Bergeron A, Bergot E, Bourdin A, et al. Voriconazole for chronic pulmonary aspergillosis: a prospective multicenter trial. Eur J Clin Microbiol Infect Dis. 2012;31:3231-9.

39. Kono Y, Tsushima K, Yamaguchi K, Kurita N, Soeda S, Fujiwara A, Sugiyama $\mathrm{S}$, et al. The utility of galactomannan antigen in the bronchial washing and serum for diagnosing pulmonary aspergillosis. Respir Med. 2013;107:1094-100. 